PROCEEDINGS OF THE

AMERICAN MATHEMATICAL SOCIETY

Volume 133, Number 4, Pages 1239-1246

S 0002-9939(04)07698-1

Article electronically published on October 18, 2004

\title{
UNCORRELATEDNESS SETS FOR RANDOM VARIABLES WITH GIVEN DISTRIBUTIONS
}

\author{
SOFIYA OSTROVSKA
}

(Communicated by Richard C. Bradley)

\begin{abstract}
Let $\xi_{1}$ and $\xi_{2}$ be random variables having finite moments of all orders. The set

$$
U\left(\xi_{1}, \xi_{2}\right):=\left\{(j, l) \in \mathbf{N}^{2}: \mathbf{E}\left(\xi_{1}^{j} \xi_{2}^{l}\right)=\mathbf{E}\left(\xi_{1}^{j}\right) \mathbf{E}\left(\xi_{2}^{l}\right)\right\}
$$

is said to be an uncorrelatedness set of $\xi_{1}$ and $\xi_{2}$. It is known that in general, an uncorrelatedness set can be arbitrary. Simple examples show that this is not true for random variables with given distributions. In this paper we present a wide class of probability distributions such that there exist random variables with given distributions from the class having a prescribed uncorrelatedness set. Besides, we discuss the sharpness of the obtained result.
\end{abstract}

\section{INTRODUCTION}

Since the notion of independence is a basic one in Probability Theory and Mathematical Statistics, various generalizations of independence have been studied. One of the earliest and most used generalizations is uncorrelatedness of random variables $\xi_{1}$ and $\xi_{2}$ defined by the condition

$$
\mathbf{E}\left(\xi_{1} \xi_{2}\right)=\mathbf{E}\left(\xi_{1}\right) \mathbf{E}\left(\xi_{2}\right),
$$

provided that all of the mathematical expectations exist. It is commonly known (e.g. [6, vol. I, p. 236) that uncorrelatedness is an essentially weaker condition than independence. In this paper we discuss problems related to uncorrelatedness of powers of random variables. We need the following definition.

Definition. Let $\xi_{1}$ and $\xi_{2}$ be random variables having finite moments of all orders. The set

$$
U\left(\xi_{1}, \xi_{2}\right):=\left\{(j, l) \in \mathbf{N}^{2}: \mathbf{E}\left(\xi_{1}^{j} \xi_{2}^{l}\right)=\mathbf{E}\left(\xi_{1}^{j}\right) \mathbf{E}\left(\xi_{2}^{l}\right)\right\}
$$

is said to be an uncorrelatedness set of $\xi_{1}$ and $\xi_{2}$.

Clearly, an uncorrelatedness set is uniquely defined for random variables with all finite moments. Obviously, if $\xi_{1}$ and $\xi_{2}$ are independent random variables, then $U\left(\xi_{1}, \xi_{2}\right)=\mathbf{N}^{2}$. Note that the converse statement is not true, as was proved in [12].

Received by the editors September 22, 2003 and, in revised form, December 22, 2003.

2000 Mathematics Subject Classification. Primary 60E05.

Key words and phrases. Uncorrelatedness, independence, uncorrelatedness set, quasianalytic class, characteristic function.

(C)2004 American Mathematical Society Reverts to public domain 28 years from publication 
Uncorrelatedness sets may be regarded as a measure of independence in the following sense: the wider an uncorrelatedness set is, the more independent random variables are. We notice that if

$$
U\left(\xi_{1}, \xi_{2}\right) \supseteq\{(j, l): j+l \leq k+1\},
$$

then random variables $\xi_{1}$ and $\xi_{2}$ are independent of degree $k$. Independence of degree $k$ was defined by C. M. Cuadras in [3] (cf. also [4]). For sequences of random variables, the uncorrelatedness condition arises in some limit theorems (cf. 7] and [8]).

Measures of independence for random variables have been intensively studied lately. Information on recent developments concerning measures and generalizations of independence can be found, for example, in [1], [5], [13], 14], [17].

It was proved in [11] that in general an uncorrelatedness set may be arbitrary. That is, for any set $U \subseteq \mathbf{N}^{2}$ there exist random variables $\xi_{1}$ and $\xi_{2}$ such that

$$
\mathbf{E}\left(\xi_{1}^{j} \xi_{2}^{l}\right)=\mathbf{E}\left(\xi_{1}^{j}\right) \mathbf{E}\left(\xi_{2}^{l}\right) \text { for all }(j, l) \in U
$$

and

$$
\mathbf{E}\left(\xi_{1}^{j} \xi_{2}^{l}\right) \neq \mathbf{E}\left(\xi_{1}^{j}\right) \mathbf{E}\left(\xi_{2}^{l}\right) \text { for all }(j, l) \in \mathbf{N}^{2} \backslash U .
$$

This result shows that uncorrelatedness of any positive integer powers of random variables does not imply uncorrelatedness of any other powers. The statement does not remain true if we prescribe distributions of random variables.

For example, for discrete random variables $\xi_{1}$ and $\xi_{2}$ taking two values, uncorrelatedness implies independence. In other words, for such random variables,

$$
U\left(\xi_{1}, \xi_{2}\right) \ni(1,1) \Rightarrow U\left(\xi_{1}, \xi_{2}\right)=\mathbf{N}^{2} .
$$

For random variables with fixed absolutely continuous distributions an uncorrelatedness set cannot be arbitrary either. Indeed, consider random variables $\xi_{1}$ and $\xi_{2}$ uniformly distributed on [0,1]. Using the Müntz Theorem (see, for example, [2] Chapter 6) one can prove that if $U\left(\xi_{1}, \xi_{2}\right)$ contains a lattice $\left\{\left(\lambda_{i}, \mu_{j}\right):(i, j) \in \mathbf{N}^{2}\right\}$, where

$$
\sum_{i=1}^{\infty} \frac{1}{\lambda_{i}}=\infty \text { and } \sum_{j=1}^{\infty} \frac{1}{\mu_{j}}=\infty
$$

then $\xi_{1}$ and $\xi_{2}$ are independent. Therefore, $U\left(\xi_{1}, \xi_{2}\right)=\mathbf{N}^{2}$.

We note that interesting examples of random variables with unusual uncorrelatedness sets are given in [15], Section 7.

In this paper we present a rather wide class of probability distributions such that there exist random variables with given distributions from the class having a prescribed uncorrelatedness set. Besides, we discuss the sharpness of the obtained result.

\section{Statement of Results}

In this paper we show that for rather slowly decreasing probability densities (however, having finite moments of all orders) and any $U \subseteq \mathbf{N}^{2}$ there exist random variables $\xi_{1}$ and $\xi_{2}$ with these densities such that $U\left(\xi_{1}, \xi_{2}\right)=U$. Our proof assumes that the densities in question satisfy the Krein condition (cf. [9]). Important examples of such densities are given in [16]. Our main result is the following statement. 
Theorem. Let $\rho_{1}(x)$ and $\rho_{2}(x)$ be probability densities having finite moments of all orders and satisfying the condition

$$
\rho_{i}(x) \geq C_{i} e^{-|x|^{\alpha}}, 0<\alpha<1(i=1,2),
$$

and let $U \subseteq \mathbf{N}^{2}$ be given.

There exist random variables $\xi_{1}$ and $\xi_{2}$ such that

i) $\xi_{1}$ and $\xi_{2}$ have absolutely continuous distributions with the densities $\rho_{1}$ and $\rho_{2}$, respectively;

ii) $U\left(\xi_{1}, \xi_{2}\right)=U$.

Remark. The condition $0<\alpha<1$ is essential and, in some sense, it cannot be improved. To be more specific, for any $\alpha \geq 1$ there exists a set $U \subset \mathbf{N}^{2}$ that is not an uncorrelatedness set for any random variables $\xi_{1}, \xi_{2}$ whose densities $\rho_{1}, \rho_{2}$ satisfy the condition

$$
\rho_{i}(x) \leq C e^{-|x|^{\alpha}}, \quad \alpha \geq 1 \quad(i=1,2) .
$$

Moreover, the statement remains true if we replace (1) with the more general condition:

$$
\mathbf{E}\left[\exp \left\{\delta\left|\xi_{i}\right|\right\}\right]<\infty \text { for some } \delta>0, i=1,2 .
$$

Indeed, let $\xi=\left(\xi_{1}, \xi_{2}\right)$ be a random vector with coordinates $\xi_{1}, \xi_{2}$ satisfying (2), characteristic function $\varphi\left(t_{1}, t_{2}\right)$ and uncorrelatedness set $U$. Assume that $\mathbf{N}^{2} \backslash U \neq \emptyset$ is finite.

We set $f\left(t_{1}, t_{2}\right):=\varphi\left(t_{1}, t_{2}\right)-\varphi\left(t_{1}, 0\right) \varphi\left(0, t_{2}\right)$. Conditions (2) imply analyticity of $f\left(t_{1}, t_{2}\right)$ in $\left\{t_{1}:\left|\operatorname{Im} t_{1}\right|<\delta / 2\right\} \times\left\{t_{2}:\left|\operatorname{Im} t_{2}\right|<\delta / 2\right\}$ (cf. [10]). Besides,

$$
\left|f\left(t_{1}, t_{2}\right)\right| \leq 2 \text { for all }\left(t_{1}, t_{2}\right) \in \mathbf{R}^{2} .
$$

Since $\mathbf{N}^{2} \backslash U$ is a nonempty finite set, $f\left(t_{1}, t_{2}\right)$ is a non-constant polynomial, which contradicts (3).

\section{Preliminaries}

In this section we introduce notation and state some results needed for the sequel. Let $\rho(x)=C_{\alpha} e^{-|x|^{\alpha}}$ be a probability density. Obviously,

$$
\rho(x) \leq a_{i} \rho_{i}(x) \text { for some } a_{i}>0(i=1,2) .
$$

Consider the function $T(r), r \geq 0$ defined by

$$
T(r)= \begin{cases}1, & \text { if } r \leq 1, \\ e^{r^{\beta}-1}, & \text { if } r>1,\end{cases}
$$

where $\beta \in(\alpha, 1)$. It can be readily seen that the function $T(r)$ satisfies the following conditions:

i) $T(r) \geq 1$

ii) $\ln T(r)$ is a convex function of $\ln r$;

iii)

$$
\int_{0}^{\infty} \frac{\ln T(r)}{r^{2}} d r<\infty
$$

Further, we apply the procedure described in [9], Ch. IV, p. 98. We set

$$
M_{n}:=\sup _{r>0} \frac{r^{n}}{T(r)} \text { for } n=0,1, \ldots
$$


We note that $M_{0}=1$. Since

$$
M_{n}=\sup _{r>0} \frac{r^{n}}{T(r)}=\sup _{r \geq 1} \frac{r^{n}}{T(r)} \leq \sup _{r \geq 1} \frac{r^{n+1}}{T(r)}=: M_{n+1},
$$

the sequence $\left\{M_{n}\right\}$ is non-decreasing.

Let us construct Ostrowski's function for the sequence $\left\{M_{n}\right\}$ :

$$
\tilde{T}(r):=\sup _{n \geq 0} \frac{r^{n}}{M_{n}} .
$$

We have

$$
\tilde{T}(r)=\frac{1}{M_{0}}=1 \text { for } r \leq 1,
$$

and it follows from the known assertion (cf. [9], Ch. IV, lemma on p. 98) that

$$
\ln \tilde{T}(r) \leq \ln T(r) \leq \ln \tilde{T}(r)+\ln r \text { for } r>1 .
$$

Inequalities (8) imply that

$$
\int_{0}^{\infty} \frac{\ln \tilde{T}(r)}{r^{2}} d r<\infty
$$

Hence by the Carleman - Ostrowski Theorem (cf., e.g. 9], Ch. IV, p. 89) the class $C_{I}\left(\left\{M_{n}\right\}\right)$ is not quasianalytic for any interval $I$.

For the sequel we need the following lemma.

Lemma 1. Let $\left\{M_{n}\right\}$ be a sequence such that the class $C_{I}\left(\left\{M_{n}\right\}\right)$ is not quasianalytic for any interval $I$. Then there exists an infinitely differentiable function $\Delta(x)$ having the following properties:

i) $\operatorname{supp} \Delta(x)=[-1,1]$;

ii) $\Delta(x)=$ const $\neq 0$ for $x \in[-1 / 2,1 / 2]$;

iii) $\Delta(x)$ is even;

iv) $\left|\Delta^{(n)}(x)\right| \leq M_{n}$ for all $n=0,1, \ldots$

Proof. Let a sequence $\left\{\underline{M}_{n}\right\}$ be the convex logarithmic regularization of $\left\{M_{n}\right\}$ (cf. [9], Ch. IV, p. 84). Note that $\left\{\underline{M}_{n}\right\}$ is non-decreasing along with $\left\{M_{n}\right\}$. We set

$$
L_{n}:=\frac{M_{n}}{2^{n}}, n=0,1,2, \ldots
$$

The class $C_{I}\left(\left\{L_{n}\right\}\right)$ is not quasianalytic for any interval $I$ (cf. [9], Ch. IV, p. 91). Therefore, there exists a function $f(x)$ such that supp $f(x) \in[-1,-1 / 2]$ and $\left|f^{(n)}(x)\right| \leq \underline{L}_{n}$, where $\underline{L}_{n}$ is the convex logarithmic regularization of $\left\{L_{n}\right\}$. The function $\varphi(x):=f^{2}(x)$ is nonnegative, supp $\varphi(x) \in[-1,-1 / 2]$ and in addition (cf. [9], p. 107)

$$
\left|\varphi^{(n)}(x)\right| \leq \underline{L}_{0} 2^{n} \underline{L}_{n} \leq \underline{L}_{0} 2^{n} L_{n}=\underline{L}_{0} \underline{M}_{n+1}, n=0,1,2, \ldots
$$

Clearly, for $\psi(x):=\varphi(x) / \underline{L}_{0}$ we have

$$
\left|\psi^{(n)}(x)\right| \leq \underline{M}_{n+1} \leq M_{n+1}, \quad n=0,1,2, \ldots
$$

Let $\tilde{\psi}(x)$ be an odd function coinciding with $\psi(x)$ on $(-\infty, 0]$. Obviously, estimate (9) is valid for $\tilde{\psi}(x)$, that is, $\left|\tilde{\psi}^{(n)}(x)\right| \leq \underline{M}_{n+1}$. We set

$$
\Delta(x):=c \int_{-1}^{x} \tilde{\psi}(t) d t
$$


where $c \in(0,1]$ is chosen in such a way that $\Delta(0) \leq M_{0}$. Evidently, the function $\Delta(x)$ satisfies the conditions $i)$ - iii) of the lemma. Since

$$
\left|\Delta^{(n)}(x)\right|=\left|c \tilde{\psi}^{n-1}(x)\right| \leq M_{n} \text { and }|\Delta(x)| \leq M_{0},
$$

we conclude that $\Delta(x)$ is a required function.

\section{Proof of the Theorem}

Let $U \subset \mathbf{N}^{2}$ be given. Consider an entire function

$$
\zeta\left(t_{1}, t_{2}\right):=\sum_{j, l=0}^{\infty} \frac{a_{j l}}{j ! l !} t_{1}^{j} t_{2}^{l}
$$

where the coefficients $a_{j l}$ are chosen in such a way that

a) $a_{j l}=0$ if $(j, l) \in U$ or $\min (j, l)=0$;

b) $a_{j l} \neq 0$ is real if $(j, l) \notin U$ and $j+l$ is even;

c) $a_{j l} \neq 0$ is imaginary if $(j, l) \notin U$ and $j+l$ is odd;

d) $\sum_{j, l=0}^{\infty}\left|a_{j l}\right|<1$.

Condition $d$ ) implies that

$$
\left|\frac{\partial^{m+n} \zeta\left(t_{1}, t_{2}\right)}{\partial t_{1}^{m} \partial t_{2}^{n}}\right| \leq 1 \text { for }\left|t_{1}\right|,\left|t_{2}\right| \leq 1 \text { and all } m, n \in \mathbf{N} .
$$

It follows from $a)$ - c) that

$$
\zeta\left(-t_{1},-t_{2}\right)=\overline{\zeta\left(t_{1}, t_{2}\right)} .
$$

Let $\left\{M_{n}\right\}$ be the numbers given by (5), and let $\Delta(t)$ be a function from Lemma 1 . We set

Note that

$$
\eta\left(t_{1}, t_{2}\right):=\zeta\left(t_{1}, t_{2}\right) \Delta\left(t_{1}\right) \Delta\left(t_{2}\right)
$$

Consider the inverse Fourier transform of $\eta\left(t_{1}, t_{2}\right)$ :

$$
\begin{aligned}
& q\left(x_{1}, x_{2}\right):=\frac{1}{(2 \pi)^{2}} \iint_{\mathbf{R}^{2}} e^{-i\left(t_{1} x_{1}+t_{2} x_{2}\right)} \eta\left(t_{1}, t_{2}\right) d t_{1} d t_{2}= \\
& \frac{1}{(2 \pi)^{2}} \int_{-1}^{1} e^{-i t_{1} x_{1}} \Delta\left(t_{1}\right) d t_{1} \int_{-1}^{1} e^{-i t_{2} x_{2}} \zeta\left(t_{1}, t_{2}\right) \Delta\left(t_{2}\right) d t_{2} .
\end{aligned}
$$

We note that the function $q\left(x_{1}, x_{2}\right)$ is real due to (11). Integrating by parts $n$ times we get

$$
\int_{-1}^{1} e^{-i t_{2} x_{2}} \zeta\left(t_{1}, t_{2}\right) \Delta\left(t_{2}\right) d t_{2}=\frac{1}{i^{n} x_{2}^{n}} \int_{-1}^{1} e^{-i t_{2} x_{2}} \frac{\partial^{n}}{\partial t_{2}^{n}}\left(\zeta\left(t_{1}, t_{2}\right) \Delta\left(t_{2}\right)\right) d t_{2} .
$$

By the Leibnitz formula,

$$
\frac{\partial^{n}}{\partial t_{2}^{n}}\left(\zeta\left(t_{1}, t_{2}\right) \Delta\left(t_{2}\right)\right)=\sum_{k=0}^{n}\left(\begin{array}{l}
n \\
k
\end{array}\right) \Delta^{(k)}\left(t_{2}\right) \frac{\partial^{n-k} \zeta\left(t_{1}, t_{2}\right)}{\partial t_{2}^{n-k}} .
$$

Therefore,

$$
q\left(x_{1}, x_{2}\right)=\frac{1}{i^{n} x_{2}^{n}} \sum_{k=0}^{n}\left(\begin{array}{l}
n \\
k
\end{array}\right) \int_{-1}^{1} e^{-i t_{2} x_{2}} \Delta^{(k)}\left(t_{2}\right) d t_{2} \int_{-1}^{1} e^{-i t_{1} x_{1}} \frac{\partial^{n-k} \zeta\left(t_{1}, t_{2}\right)}{\partial t_{2}^{n-k}} \Delta\left(t_{1}\right) d t_{1} .
$$


Using $m$ times integration by parts and the Leibnitz formula, we obtain

$$
\begin{aligned}
\int_{-1}^{1} & e^{-i t_{1} x_{1}} \frac{\partial^{n-k} \zeta\left(t_{1}, t_{2}\right)}{\partial t_{2}^{n-k}} \Delta\left(t_{1}\right) d t_{1} \\
& =\frac{1}{i^{m} x_{1}^{m}} \int_{-1}^{1} e^{-i t_{1} x_{1}} \frac{\partial^{m}}{\partial t_{1}^{m}}\left(\frac{\partial^{n-k} \zeta\left(t_{1}, t_{2}\right)}{\partial t_{2}^{n-k}} \Delta\left(t_{1}\right)\right) d t_{1} \\
& =\frac{1}{i^{m} x_{1}^{m}} \sum_{j=0}^{m}\left(\begin{array}{c}
m \\
j
\end{array}\right) \int_{-1}^{1} e^{-i t_{1} x_{1}} \frac{\partial^{m-j+n-k} \zeta\left(t_{1}, t_{2}\right)}{\partial t_{1}^{m-j} \partial t_{2}^{n-k}} \Delta^{(j)}\left(t_{1}\right) d t_{1} .
\end{aligned}
$$

Therefore,

$$
\begin{array}{r}
q\left(x_{1}, x_{2}\right) \\
=\frac{1}{i^{m+n} x_{1}^{m} x_{2}^{n}} \sum_{k=0}^{n} \sum_{j=0}^{m}\left(\begin{array}{l}
n \\
k
\end{array}\right)\left(\begin{array}{c}
m \\
j
\end{array}\right) \int_{-1}^{1} \int_{-1}^{1} e^{-i\left(t_{1} x_{1}+t_{2} x_{2}\right)} \Delta^{(j)}\left(t_{1}\right) \Delta^{(k)}\left(t_{2}\right) \\
\times \frac{\partial^{m-j+n-k} \zeta\left(t_{1}, t_{2}\right)}{\partial t_{1}^{m-j} \partial t_{2}^{n-k}} d t_{1} d t_{2}
\end{array}
$$

for all $m, n \in \mathbf{N}$.

We may estimate the modulus of $q\left(x_{1}, x_{2}\right)$ using (10) and Lemma 1(iv) as follows:

$$
\begin{gathered}
\left|q\left(x_{1}, x_{2}\right)\right| \leq \frac{4}{\left|x_{1}\right|^{m}\left|x_{2}\right|^{n}} \sum_{k=0}^{n} \sum_{j=0}^{m}\left(\begin{array}{l}
n \\
k
\end{array}\right)\left(\begin{array}{c}
m \\
j
\end{array}\right) M_{j} M_{k} \\
\leq \frac{4 M_{m} M_{n}}{\left|x_{1}\right|^{m}\left|x_{2}\right|^{n}} \sum_{k=0}^{n} \sum_{j=0}^{m}\left(\begin{array}{l}
n \\
k
\end{array}\right)\left(\begin{array}{c}
m \\
j
\end{array}\right)=4 \frac{2^{m} M_{m}}{\left|x_{1}\right|^{m}} \cdot \frac{2^{n} M_{n}}{\left|x_{2}\right|^{n}}=4 \frac{M_{m}}{\left|\frac{x_{1}}{2}\right|^{m}} \cdot \frac{M_{n}}{\left|\frac{x_{2}}{2}\right|^{n}}
\end{gathered}
$$

for all $m, n \in \mathbf{N}$.

Hence

$$
\left|q\left(x_{1}, x_{2}\right)\right| \leq 4 \inf _{m, n} \frac{M_{m}}{\left|\frac{x_{1}}{2}\right|^{m}} \cdot \frac{M_{n}}{\left|\frac{x_{2}}{2}\right|^{n}}=4 \frac{1}{\tilde{T}\left(\frac{\left|x_{1}\right|}{2}\right)} \frac{1}{\tilde{T}\left(\frac{\left|x_{2}\right|}{2}\right)},
$$

where $\tilde{T}(r)$ is given by (6). It follows from (7) and (8) that

$$
\tilde{T}(r)=1 \text { for } r \leq 1 \text { and } \tilde{T}(r) \geq \frac{T(r)}{r} \text { for } r>1 .
$$

Hence

$$
\left|q\left(x_{1}, x_{2}\right)\right| \leq 4 \frac{\left|x_{1}\right|\left|x_{2}\right|}{T\left(\frac{\left|x_{1}\right|}{2}\right) T\left(\frac{\left|x_{2}\right|}{2}\right)} \leq C \omega\left(x_{1}\right) \omega\left(x_{2}\right),
$$

where $C$ is a positive constant and

$$
\omega(x):= \begin{cases}|x|, & \text { if }|x| \leq 2, \\ |x| \exp \left(-\left|\frac{x}{2}\right|^{\beta}\right), & \text { if }|x|>2 .\end{cases}
$$

Since $\alpha<\beta$, this implies that $\varepsilon\left|q\left(x_{1}, x_{2}\right)\right| \leq \rho\left(x_{1}\right) \rho\left(x_{2}\right)$ for $\varepsilon>0$ small enough. It follows from (4) that

$$
\varepsilon\left|q\left(x_{1}, x_{2}\right)\right| \leq a_{1} a_{2} \rho_{1}\left(x_{1}\right) \rho_{2}\left(x_{2}\right) .
$$

We set

$$
f\left(x_{1}, x_{2}\right):=\rho_{1}\left(x_{1}\right) \rho_{2}\left(x_{2}\right)+\delta q\left(x_{1}, x_{2}\right),
$$

where $\delta \in\left(0, \varepsilon / a_{1} a_{2}\right)$. Clearly, for this choice of $\delta$ we have $f\left(x_{1}, x_{2}\right) \geq 0$. 
We show that $f\left(x_{1}, x_{2}\right)$ is a probability density. Indeed, the definition of $q\left(x_{1}, x_{2}\right)$ given by (13) implies that

$$
\eta\left(t_{1}, t_{2}\right)=\iint_{\mathbf{R}^{2}} e^{i\left(t_{1} x_{1}+t_{2} x_{2}\right)} q\left(x_{1}, x_{2}\right) d x_{1} d x_{2} .
$$

Hence by (12)

$$
\iint_{\mathbf{R}^{2}} q\left(x_{1}, x_{2}\right) d x_{1} d x_{2}=\eta(0,0)=0,
$$

and, therefore,

$$
\iint_{\mathbf{R}^{2}} f\left(x_{1}, x_{2}\right) d x_{1} d x_{2}=\iint_{\mathbf{R}^{2}} \rho_{1}\left(x_{1}\right) \rho_{2}\left(x_{2}\right) d x_{1} d x_{2}+\delta \iint_{\mathbf{R}^{2}} q\left(x_{1}, x_{2}\right)=1 .
$$

Thus, $f\left(x_{1}, x_{2}\right)$ is a probability density.

Let $\xi=\left(\xi_{1}, \xi_{2}\right)$ be a random vector having absolutely continuous distribution with the density $f\left(x_{1}, x_{2}\right)$. The characteristic function of the vector $\xi$ equals

$$
\varphi\left(t_{1}, t_{2}\right)=\psi\left(t_{1}, t_{2}\right)+\delta \eta\left(t_{1}, t_{2}\right),
$$

where $\psi\left(t_{1}, t_{2}\right)$ is a characteristic function of a random vector with independent coordinates whose densities are $\rho_{1}$ and $\rho_{2}$. Since by (12) we have $\eta\left(t_{1}, 0\right)=\eta\left(0, t_{2}\right)=$ 0 , it follows that

$$
\varphi\left(t_{1}, 0\right)=\psi\left(t_{1}, 0\right) ; \quad \varphi\left(0, t_{2}\right)=\psi\left(0, t_{2}\right) .
$$

This means that the coordinates $\xi_{1}$ and $\xi_{2}$ have absolutely continuous distributions with the given densities $\rho_{1}$ and $\rho_{2}$.

Besides,

$$
\begin{array}{r}
\left.\frac{\partial^{j+l}}{\partial t_{1}^{j} \partial t_{2}^{l}} \varphi\left(t_{1}, t_{2}\right)\right|_{(0,0)}=\left.\frac{\partial^{j+l}}{\partial t_{1}^{j} \partial t_{2}^{l}} \psi\left(t_{1}, t_{2}\right)\right|_{(0,0)}+\left.\delta \frac{\partial^{j+l}}{\partial t_{1}^{j} \partial t_{2}^{l}} \eta\left(t_{1}, t_{2}\right)\right|_{(0,0)} \\
=\left.\frac{\partial^{j+l}}{\partial t_{1}^{j} \partial t_{2}^{l}} \varphi\left(t_{1}, 0\right) \varphi\left(0, t_{2}\right)\right|_{(0,0)}+\delta a_{j l}=\left.\frac{\partial^{j+l}}{\partial t_{1}^{j} \partial t_{2}^{l}} \psi\left(t_{1}, 0\right) \psi\left(0, t_{2}\right)\right|_{(0,0)}+\delta a_{j l} .
\end{array}
$$

Therefore,

$$
\mathbf{E}\left(\xi_{1}^{j} \xi_{2}^{l}\right)=\mathbf{E}\left(\xi_{1}^{j}\right) \mathbf{E}\left(\xi_{2}^{l}\right)+\delta a_{j l} .
$$

Since $a_{j l}=0$ for $(j, l) \in U$ and $a_{j l} \neq 0$ for $(j, l) \in \mathbf{N}^{2} \backslash U$, it follows that

$$
\mathbf{E}\left(\xi_{1}^{j} \xi_{2}^{l}\right)=\mathbf{E}\left(\xi_{1}^{j}\right) \mathbf{E}\left(\xi_{2}^{l}\right) \text { for }(j, l) \in U
$$

and

$$
\mathbf{E}\left(\xi_{1}^{j} \xi_{2}^{l}\right) \neq \mathbf{E}\left(\xi_{1}^{j}\right) \mathbf{E}\left(\xi_{2}^{l}\right) \text { for }(j, l) \in \mathbf{N}^{2} \backslash U .
$$

Thus, $U$ is an uncorrelatedness set for $\xi_{1}$ and $\xi_{2}$.

\section{ACKNOWLEDGEMENTS}

I would like to express my gratitude to the anonymous referee for his/her thorough reading of the manuscript and valuable suggestions, and to Dr. Paul Danesh for his help in preparation of the manuscript. 


\section{REFERENCES}

[1] V. Benes, J. Stepan, (eds.) (1997) Distributions with Given Marginals and Moment Problems. Kluwer Academic Publishers MR 1614650 (98k:60003)

[2] E.W. Cheney (1982) Introduction to Approximation Theory. $2^{\text {nd }}$ Ed. Chelsea Publishig Company, N.Y. MR1656150 (99f:41001)

[3] C. M. Cuadras, Theoretical, experimental foundations and new models of factor analysis, Investigación Pesquera, 39 (1972), pp. 163-169 (in Spanish)

[4] C. M. Cuadras, First principal component characterization of a continuous random variable, Universitat de Barcelona, Institut de Matemàtica, Mathematics Preprint Series, No 327 (2003)

[5] G. Dall'Aglio, S. Kotz, G. Salinetti, (eds.) (1991) Advances in Probability Distributions with Given Marginals. Kluwer Academic Publishers. MR1215942 (93k:60004)

[6] W. Feller, An Introduction to Probability Theory and Its Applications. Wiley, New-York, 1986

[7] A. Jakubowski, S. Kwapień, On multiplicative systems of functions, Bull. Acad. Polon. Sci. Sr. Sci. Math. 27 (1979), (9), pp. 689-694. MR0600722 (82c:60014)

[8] J. Komlòs, A central limit theorem for multiplicative systems. Canad. Math. Bull., 16 (1973), pp. 67-73. MR0324753(48:3102)

[9] P. Koosis, The Logarithmic Integral, Vol. I, Cambridge: Cambridge University Press, 1988. MR 0961844 (90a:30097)

[10] Ju. V. Linnik, I. V. Ostrovskii, Decomposition of Random Variables and Vectors, AMS, Providence, R. I., 1977. MR0428382 (55:1404)

[11] S. Ostrovska, Uncorrelatedness and correlatedness of powers of random variables, Arch. der Math. 79 (2002), pp. 141-146. MR.1925381 (2003g:60026)

[12] S. Ostrovska, A Scale of Degrees of Independence of Random Variables. Indian J. of Pure and Applied Math., 29 (1998), (5), pp. 461-471. MR.1627847 (99i:60029)

[13] L. Rüschendorf, B. Schweitzer, M. D. Taylor (eds.) Distributions with Fixed Marginals and Related Topics. Institute of Mathematical Statistics. Lecture Notes - Monograph Series, Hayward, California, 1996. MR,1485518 (98g:60004)

[14] J. Stoyanov, Dependency Measure for Sets of Random Events or Random Variables. Statist. E Prob. Letters (1995) 23, pp. 13-20. MR1333372 (96c:60003)

[15] J. Stoyanov, Counterexamples in Probability. 2nd Ed. Wiley, Chichester - New York, 1997 MR0930671 (89f:60001)

[16] J. Stoyanov, Krein condition in probabilistic moment problems, Bernoulli 6, No.5(2000), 939-949. MF 1791909 (2001i:44014)

[17] J. Stoyanov, Sets of Binary Random Variables with a Prescribed Independence/Dependence Structure. The Mathematical Scientist, vol. 28, no. 1(2003), 19-27. MF1995161

Department of Mathematics, Atilim University, 06836 Incek, Ankara, Turkey

E-mail address: ostrovskasofiya@yahoo.com 